Case Report

\title{
Leprosy in a Patient Coinfected with HIV and Syphilis
}

\author{
${ }^{1,2}$ Carla Andréa Avelar Pires, ${ }^{2}$ Julius Caesar Mendes Soares, ${ }^{1}$ Maria Amélia Lopes dos Santos, ${ }^{4}$ Herbert \\ Paulino Cordeiro, ${ }^{3}$ Victoria Juliana Campos Lodi, ${ }^{3}$ Raphael Pereira do Couto Rocha, ${ }^{1}$ Alianne Violet Alves \\ Chichester and ${ }^{1}$ Francisca Regina Oliveira Carneiro
}

\author{
${ }^{1}$ Department of Dermatology, Universidade do Estado do Pará, Belém, Pará, Brazil \\ ${ }^{2}$ Núcleo de Medicina Tropical da Universidade Federal do Pará, Brazil \\ ${ }^{3}$ Department of Dermatology, Centro Universitário do Estado do Pará, Belém, Pará, Brazil \\ ${ }^{4}$ Department of Infectology, Faculdade Metropolitana da Amazônia, Belém, Pará, Brazil
}

\author{
Article history \\ Received: 04-03-2019 \\ Revised: 23-05-2019 \\ Accepted: 23-07-2019 \\ Corresponding Author: \\ Carla Andréa Avelar Pires \\ Department of Dermatology, \\ Universidade do Estado do Pará, \\ Belém, Pará, Brazil \\ Email: carlaavelarpires@gmail.com
}

\begin{abstract}
Case report of a male patient, fifty years old, born in the Amazon region of Belém, Pará, Northern Brazil. He was attended at a University service of reference in Dermatology on 09/14/2018, referring to "ball injuries" about a year ago with a sudden and progressive appearance in the abdomen with dissemination to the whole body. Three months ago he was diagnosed as a person living with the HIV Virus (PLHIV) and Antiretroviral Therapy (ART) with TDF + 3TC + DTG was started and VDRL positive (1/256) already under treatment with Benzathine penicillin. Physical examination revealed the presence of erythematous-infiltrated nodules, some of which were confluent and normochromic and were disseminated in the auricle, face, trunk, upper limbs and in a smaller number in the lower limbs. The patient was confused in the thermal sensitivity test and complained of numbness. Under suspicion of leprosy, smear microscopy of skin with 5+ positive result and histopathological biopsy of the skin lesion. Patient was also diagnosed with Secondary Syphilis and Leprosy clinical form Virchowiana, treatment with multibacillary specific multidrugresistant chemotherapy (MDT-MB) allied to prednisone at the inicial dose $60 \mathrm{mg}$. This case shows a coinfection, Human Immunodeficiency Virus (HIV), Leprosy and Syphilis, the relation of HIV-Leprosy and the influence of Syphilis on this coinfection, changing the clinical form and their evolutions.
\end{abstract}

Keywords: Leprosy, HIV, Syphilis, Coinfection

\section{Introduction}

Leprosy is a chronic dermatologic infection that has Mycobacterium leprae as a ethiological agent. This issue still affects millions of people, especially, in underdeveloped countries. In 2017, 150 countries, 6 regions of the world were described by World Health Organization (WHO) according to the epidemiology of leprosy: 41 countries in the African Region (AFR), 33 in the Region of the Americas (AMR) (WHO, 2016). The statistics in Brazil, presented a new cases rate of 26,875, with higher incidence in the Central West $(33,84)$, North $(28,82)$ and Northeast $(20,58)$ regions (Active Registry: Number and Percentage, 2017).

The disease manifests a spectrum of clinic pathologic forms, ranging from Tuberculoid Leprosy (TL), borderline forms, to Lepromatous Leprosy (LL) and can be paucibacillary or multibacillary in skin lesions. The tuberculoid type has a good recall of cell-mediated immune by Th1 cytokinesinlesions, anothertype, lepromatous.
Coinfections between Human Immunodeficiency Virus (HIV) and bacteria in developed and Underdeveloped countries started to have a huge impacton public health. In the first contact, scientists created the theory that AIDS could change the disease of leprosy, in higher cases of multibacillary, but nowadays, the recently studies shows the opposite, a higher increase of cases paucibacillary, with no change in the way of this disease, without drug interactions (Lockwood and Lambert, 2010; Pires et al., 2015). The histopathological observations reveal a normal spectrum of leprosy biopsies from coinfected patients, even among those with advanced immunodeficiency (Pires et al., 2015).

Patients with leprosy - HIV coinfection do not present change in immunological, histological and clinical course of the disease, therefore, there is no need for different treatment management (Ridley and Jopling, 1966; Hussain et al., 2005). However, because of immunodeficiency, HIVpositive patients are more likely to contract syphilis, making treatment more difficult (Nelson, 2005). 
The clinical characteristics shared by leprosy and syphilis make clinical diagnosis a major challenge. Cutaneous manifestations are the most frequent signs and can occur in several forms of dermatological lesions (nodules, plaques, papules, infiltrations, etc). Because of these common characteristics and nonspecific lesions between both diseases the differential diagnosis is impaired (Romanini et al., 2005).

This case report shows a patient with a coinfection Human Immunodeficiency Virus (HIV), Leprosy and Syphilis that has a very well response to Antiretroviral Therapy (ART), Multidrug-resistant Multibacillary (MDT-MB) and Penicillin Benzatine Intramuscular.

\section{Case Report}

A Male patient, fifty years old, born in the Amazon region of Belém, Pará, northern Brazil. He lives alone and was attended at a University service of reference in Dermatology on 09/14/2018, referring to "ball injuries" (Fig. 3) about a year ago with a sudden and progressive appearance in the abdomen with dissemination to the whole body. Three months ago he was diagnosed as a person living with the HIV virus (PLHIV) and Antiretroviral Therapy (ART) with TDF + 3TC + DTG was started, with a viral load of 95,063 copies, CD4 + of 372 cells/mm3 and VDRL positive (1/256) already under treatment with Benzatine penicillin. Physical examination revealed the presence of erythematous-infiltrated nodules, some of which were confluent and normochromic and were disseminated in the auricle, face, trunk, upper limbs and in a smaller number in the lower limbs. The patient was confused in the thermal sensitivity test and complained of numbness in the right hand, where weakness of the fifth finger was observed in the monofilament test (violet color), ipsilateral ulnar nerve thickening, edema in lower limbs, decreased left foot force and monofilament sensitivity lilac on both feet. Under suspicion of leprosy, smear microscopy of skin with 5+ positive result and histopathological biopsy of the skin lesion (Fig. 1 and 2). In addition to these tests, it also shows results of Toxoplasmosis IgG reagent, Cytomegalovirus reagent, Fluorescent Treponemal Antibody Absortion (FTA-ABS) reagent, Total unreacted Anti-HBc, Non-reactive Anti-HBs. Patient was also diagnosed with Secondary Syphilis and Leprosy clinical form Virchowian, treatment with multibacillary specific multidrug-resistant chemotherapy (MDT-MB) allied to prednisone at the initial dose $60 \mathrm{mg}$, with a reduction 5 by 5 mg, 15 by 15 days until the total regretion. At the time of the return visit the patient brought a daughter who was diagnosed with a leprosy lesion clinically tuberculoid form.

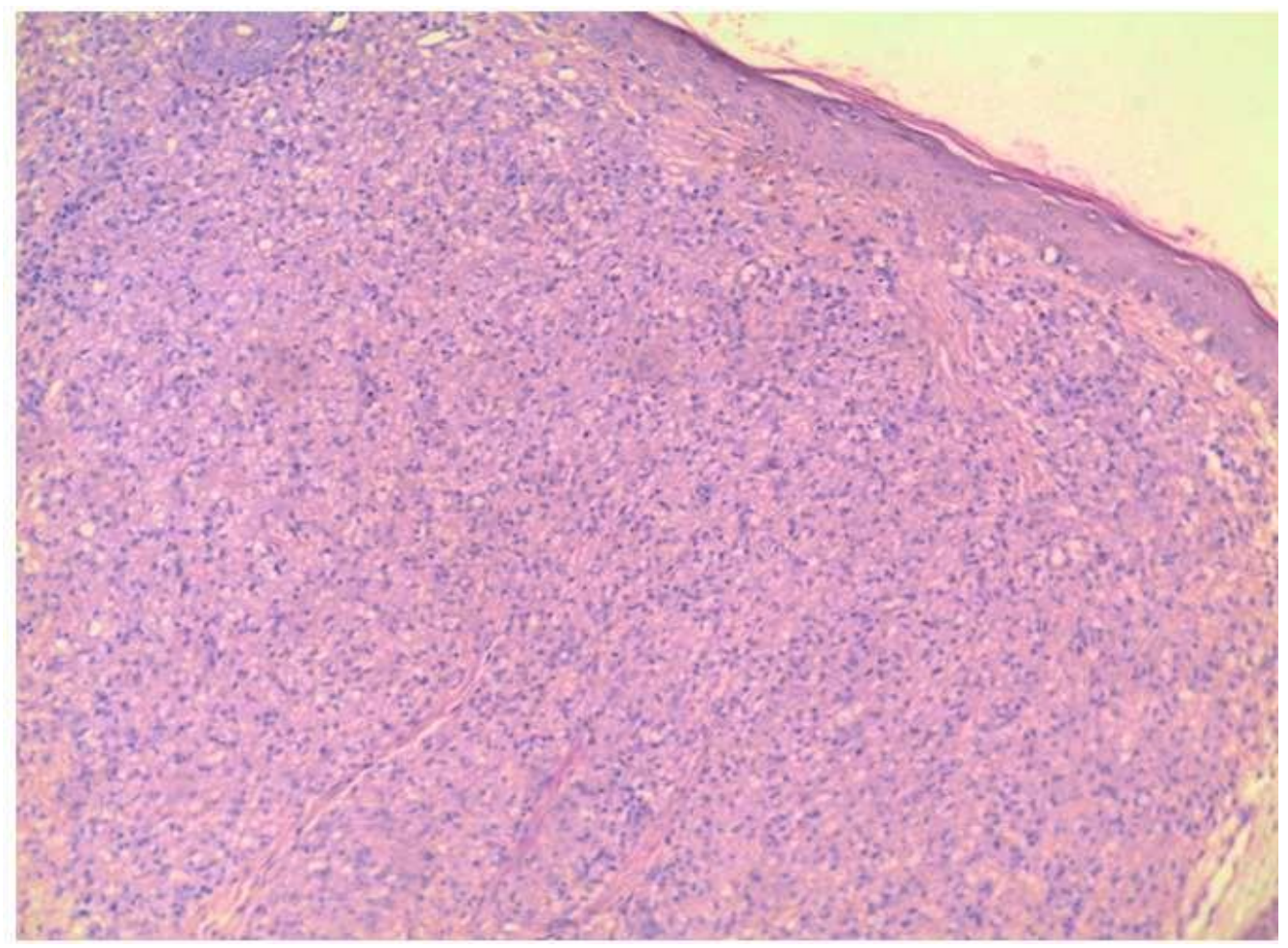

Fig. 1: Epidermis with no changes,Unna track and a higher quantity of bacilli. (Hematoxilyn-eosin, HE) 


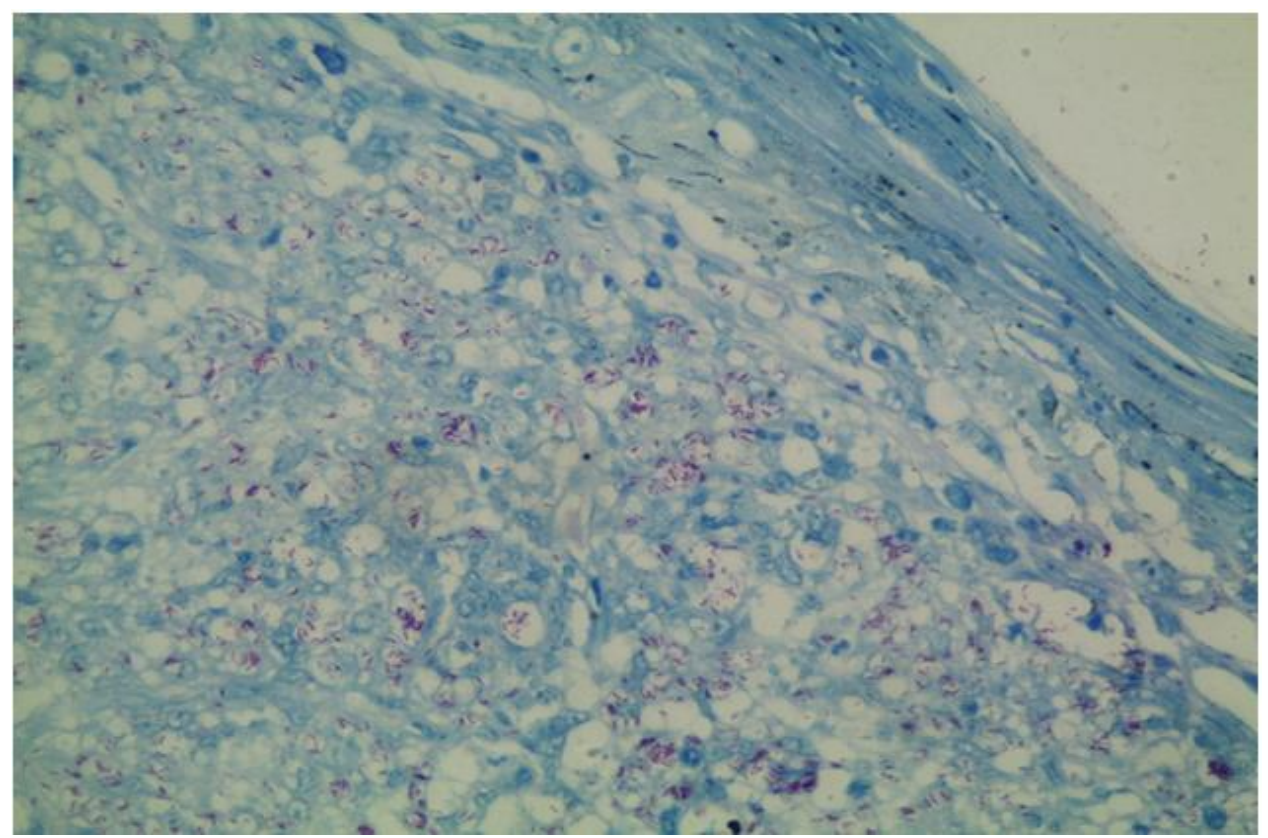

Fig. 2: Extensive dermal infiltrate rich in foamy macrophages and bacilli (Fite-Faraco technique, original magnification, 40x)
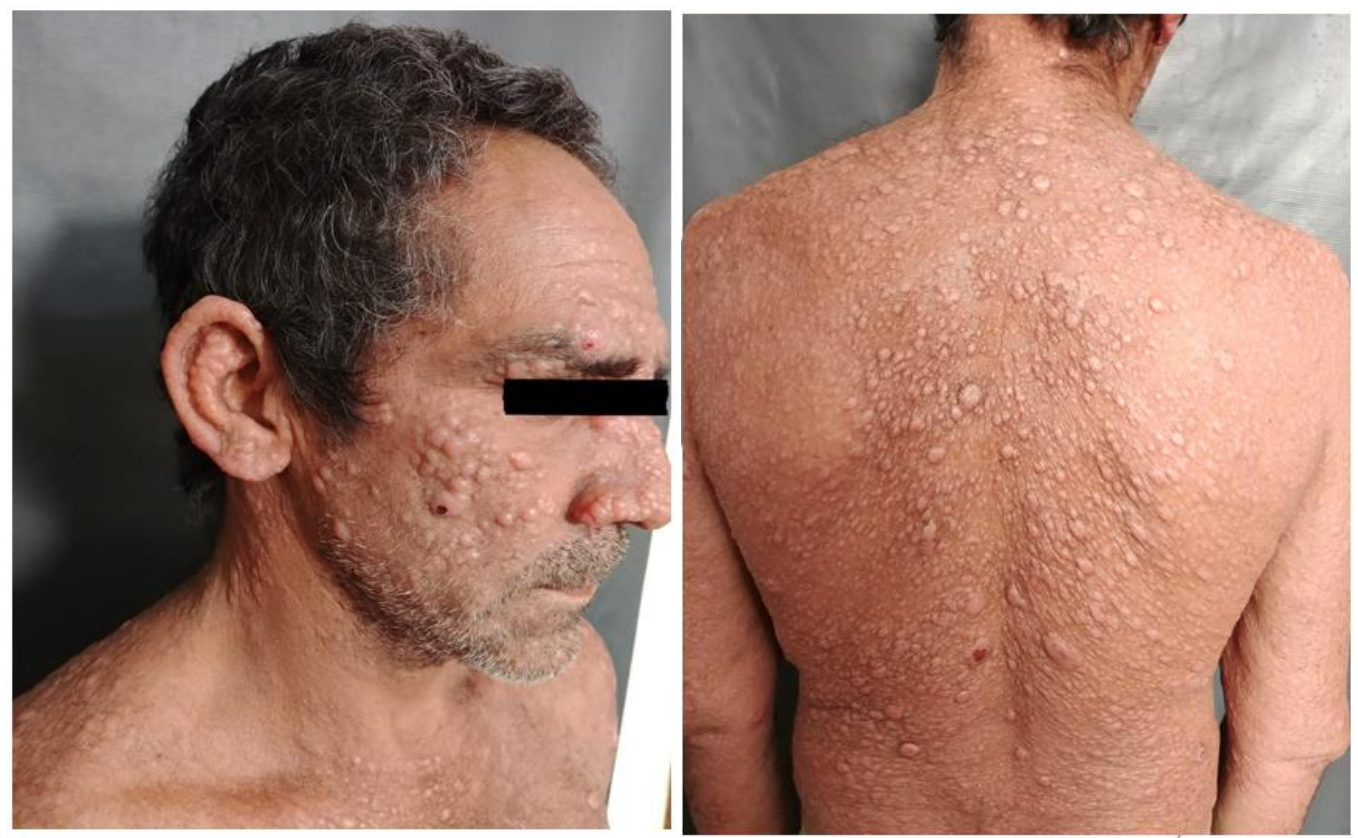

Fig. 3: Erythematous-infiltrated nodules, some of which were confluent and normochromic and were disseminated in the auricle, face, trunk, upper limbs and in a smaller number in the lower limbs. This images shows the day of the diagnosis

The present study is a case report of HIV patient coinfected with Leprosy and Syphilis. The diagnosis of leprosy and syphilis was done in a medical service at the Dermatology Service of the State University of Pará. The patient came with previous diagnosis of HIV made by a referral center in infectious diseases already in use of antiretrovirals.
Tests performed for the diagnosis of leprosy: Skin test of thermal sensitivity showing reduction in some points and in others the patient was confused. In the neurological examination the patient presented a loss of sensation in the left hand where he did not feel the green or blue monofilament and there was evidence of thickening of the ulnar nerve on the same side. A biopsy was performed 
with histopathological skin lesion with hematoxylin and eosin staining, as well as Fite-Faraco showing abundant bacilli and numerous foamy macrophages.

\section{Other Tests Performed}

Serologies for Toxoplasmosis, Cytomegalovirus, Hepatitis B, treponemal test FTA-ABS and nontreponemal VDRL were requested. The IgG antibodies were positive for Toxoplasmosis and Cytomegalovirus; FTA-ABS and VDRL were also reagent (1/256)

Sample: 1 patient, due to the uniqueness of the infections and the forms they presented.

\section{Discussion}

The disease caused by infection with Human Immunodeficiency Virus (HIV) generates a dysfunction of the immune system and the natural evolution of the disease is characterized by the reduction of TCD4 + immune cells (Tarique et al., 2017). Comes in different phases of the course of the disease as the stage of acute infection, asymptomatic infection (latency ranging from 3 to 10 years) and symptomatic disease, considered to severe stage of the disease, marked by severe symptoms of disease, such as prolonged fever, night sweats, significant weight loss, weakness and enlarged lymph nodes and opportunistic infections such as tuberculosis, pneumonia, toxoplasmosis, candidiasis, non-Hodgkin's lymphomas and Kaposi's sarcoma (Tarique et al., 2017). The HIV infection is defined when it is performed rapid testing and the result is reactive, after that must be performed the confirmatory molecular Western Blot test (Brazil, 2016). The first places where the disease has been diagnosed in the United States, Haiti and Central Africa, the end of the 1970s, only in 1980 was diagnosed and notified in Brazil today is considered an epidemic in the country (Pires et al., 2015).

In the diagnosis of syphilis, a flowchart should be followed, first a non-treponemal test is performed (more commonly the Veneral Disease Research LaboratoryVDRL) and then, if positive, the treponemal test, the Fluorescent Treponemal Antibody Absorption TestFTA-ABS, one of the most used. The disease is caused by the bacterium Treponema Pallidum that can manifest in a primary form through a single, painless, non-itchy lesion in the genital region, mouth or skin, usually 10 to 90 days after infection; secondary when there are several lesions, spots on the body, including palms and plants, along with symptoms such as fever, malaise, appearing about 6 weeks to 6 months after the initial lesion; there is still the latent phase, usually asymptomatic; and finally, the tertiary that can manifest between 2 and 40 years after the onset of infection, with systemic and severe presentations (Brazil, 2016).
The diagnosis of leprosy is most often clinical, marked by the alteration of sensitivity (thermal, tactile and painful) in the cutaneous lesions and there may still be changes of strength/sensitivity in pathways of peripheral nerves and when necessary histopathological can be used : in which one can observe lymphocytes and histiocytes with slight infiltrate, perineural or periaxial (indeterminate); presence of histiocytes or macrophages differentiate into epithelioid cells forming a granuloma, in intimate contact with the epidermis (tuberculoid); presence of macrophages filled with bacilli and globules, presenting a vacuolar cytoplasm with lipid degeneration (Virchow form- Virchowiana cell); lymphocytes in dimorfa-virchowiana and are less frequent in VirchowianaVirchowiana; there is also the classification of histioides, atypical multibacillary form, with fusiform, xanthomized and intertwined histiocytes containing innumerable bacilli in their interior (Ridley and Jopling, 1966).

Several studies, observed the interference of HIV and Syphilis one over the other, altering the evolution of diseases. In particular, due to shortening the time of onset of primary and secondary syphilis, often with overlapping cases, in addition to the rapid evolution to tertiary syphilis, with severe neurological repercussions such as neurosyphilis. It is recommended the complete treatment of Highly Active Antiretroviral Therapy (HAART), together with the administration of antibiotic therapy (intramuscular benzathine penicillin 7,200,000 IU) (Brazil, 2016). In another study conducted in 2016, it was shown that both diseases share of the same period of transmission, the period of immunological window of Syphilis predisposes to HIV, by increase of receptors cells receiving the virus, increase in the number of its receptors, in a total of 264 patients, 90 presented coinfection. An observation made in this study was that in 38 of the 90 patients, it presented negative VDRL and positive FTAABS, which led to the conclusion that in the case of this coinfection the best marker for confirmation is FTA-ABS (Teresinha Boni Ribeiro and Jacociunas, 2016).

Syphilis is eight times more prevalent in individuals with the HIV virus compared to the general population and this coinfection can change the natural story of the disease, since they may present treatment failures in addition to more frequent neurological changes. Another factor is the presence of altered manifestation of recent syphilis, latent and late, leading to a tendency to severity of symptoms and injuries, taking care with neurological changes, as neurosyphilis in severe form (Secretary of Department of STD, 2014).

According to the World Health Organization, America is one of the continents that has relevant infection rates for Mycobacterium leprae. In Brazil, studies were specifically carried out to analyze HIV and Leprosy coinfection, showing a greater occurrence in males, in the age group 31 to 59 years, with the most common operational 
classification being the paucibacillary and the most common clinical forms were the Boderline Tuberculoid, Boderline boderline and Tuberculoid. In addition, the incidence of leprosy reaction in this group presented more common type I or reverse reaction, with a moderate character (Pires et al., 2015).

In the cases described with co-infection Leprosy and HIV, it cannot confirm that HIV changes the evolution of leprosy disease. In fact, most studies show that there is no change in the disease (Couppie et al., 2004; Sarno et al., 2008). A cohort study of 40 patients in 2015 in Brazil, found that coinfected patients with leprosy reaction, their total use of the Antiretro Viral Therapy (ART) and most of them presenting the Immune Reconstitution Syndrome. An interesting and contradictory fact, which still requires a great deal of study, is leprosy in its paucibacillary forms presenting a predominance of immune response mediated by lymphocytes with Th1 and Th2 profiles in the multibacillary, the latter being more severe. However, the prevalence of HIV/leprosy carrier is most often with paucibacillary forms, presenting leprosy in your soft form (Pires et al., 2015).

The human immunodeficiency virus after accomplish important modifications in the individual's immune system favors coinfections with other viruses. Studies from Brazil have shown that many leprosy patients have positive markers for hepatitis B and hepatitis C (Rosa et al., 1992; Ramos et al., 2011). The clinical form of leprosy developed by patients coinfected with hepatitis $\mathrm{C}$ was Virchowian form, acquiring a more severe course for both diseases, without concrete explanations (Determinaçãoda Prevalênciade Coinfecçõespelos Vírus da Imunodeficiência Humana, 2005).

Access to Anti Retrovirus Therapy was guaranteed by the Brazilian Government and by some national institutions, which allowed the disease to change from being lethal to a chronic, controlled disease. The universal availability of effective medicines and the adequate clinical monitoring have increased both the expectation and the quality of life of people living with HIV/AIDS, bringing new challenges for the promotion of integral care of these people, as well as new coinfections emerged that earlier, of the more effective antiretroviral therapy, were not seen observed because to high early mortality (Brasil, 2015).

\section{Conclusion}

The present case is a patient with multiple infectionsHIV in AIDS, secondary syphilis and Virchowian leprosy-that has acquired an exuberant clinical aspect. This is in contradiction with most of the literature describing HIV-positive patients developing softer clinical forms of leprosy (Teresinha Boni Ribeiro and Jacociunas, 2016). However, it must be considered that it is a patient presenting with several concomitant infections which may be causing immune dysregulation leading to clinical manifestations were more exacerbated and difficult to control, like neurites, even though these were mediated by $\mathrm{T}$ cells. In addition to the above, the patient still presented oscillations in the value of VDRL even the patient denying epidemiology of reinfection. A cerebrospinal fluid test was performed with normal results. A new treatment cycle with benzathine penicillin was performed at the same previous dose, resulting in reduction of VDRL. Also noteworthy is the importance of the examination of communicants, where the daughter diagnosed and treated also for leprosy, marks the breakdown of the transmission chain of this disease. Thus, the patient presented an evolution of the leprosy disease, proving that the presence of HIV-leprosy coinfection alone did not interfere in the course of both diseases, but by adding another pathology to the group, in the case of syphilis and Hepatitis B, may lead to immune dysregulation leading to an exacerbated cellular response in HIV, leprosy, syphilis and hepatitis B infections. This study exhibited that leprosy and HIV has a different and independent course, but the coinfection with another diseases could cause exuberant clinical aspect of leprosy.

\section{Acknowledgement}

A great and specially acknowledgment to the Dr. Julius Caesar Mendes Soares Monteiro for sent this patient to the Ambulatory of Dermatology in Universidade Federal do Pará and for inviting us to study this exhuberant case.

\section{Author's Contributions}

Alianne Violet Alves Chichester: Assisted in acquisition of data auxiliar.

Carla Andrea AvelarPires: Drafted the manuscript.

Francisca Regina Oliveira Carneiro: Made the final review of the manuscript, critically.

Herbert PaulinoCordeiro: Acquisition of data.

Julius Caesar Mendes Soares Monteiro: Organized the data in detail.

Maria Amelia Lopes Santos: Histopathological biopsy analysis.

Raphael Pereira do Couto Rocha: Responsible for project edition.

Victoria Juliana Campos Lodi: Made the liter review and revision of the manuscript. 


\section{Compliance with Ethical Standards}

\section{Funding}

The authors declare that they have received no funds.

\section{Conflicts of Interest}

The authors declare that they have no conflict of interest.

\section{Ethical Approval}

All procedures performed in studies involving human participants were in accordance with the ethical standards of the Universidade do Estado do Pará ethic committee and with the 1964 Helsinki declaration and its later amendments or comparable ethical standards.

\section{Informed Consent}

Informed consent was obtained from this participant, included in the study. Additional informed consent was obtained for whom identifying information is included in this article.

\section{References}

Active Registry: Number and Percentage, 2017. New cases of leprosy: Number, percentage and coefficient, age group, operational classification, sex, degree of incapacity, contacts examined, by states and regions, Brazil.

Brazil, 2015. Ministry of Health. The Management of HIV Infection in Basic Care: A Handbook for Medical Professionals [internet]. Brasília, DF: Ministry of Health; 2015

Brazil, 2016. Ministry of Health. Secretariat of Health Surveillance. Epidemiological Bulletin Syphilis.

Couppie, P., S. Abel, H. Voinchet, M. Roussel and R. Helenon et al., 2004. Immune reconstituition inflammatory syndrome associated with HIV and Leprosy. Arch Dermatol, 140: 997-1000. DOI:10.1001/archderm.140.8.997

Determinação da Prevalência de Coinfecções pelos Vírus da Imunodeficiência Humana, 2005. Hepatite B, Hepatite C e Linfotrópico de Células T Humanas em Pacientes Portadores de Hanseníase/Anna Carolina de Moraes Braga - Curitiba.

Hussain, T, S. Sinha, K.K. Kulshreshtha, K. Katoch and V.S. Yadav et al., 2005. Seroprevalence of HIV infection among leprosy patients in Agra, India: trends and perspective. Int $\mathbf{J}$ Lepr Other Mycobact Dis. 73: 93-99.

Lockwood, D.N.J. and S.M. Lambert, 2010. Leprosy and HIV, where are we at? Lepr Rev., 81: 169-175.
Nelson, K.E., 2005. Leprosy and HIV infection (rarely the twain shall meet?). Int. J. Lepr. Other. Mycobact. Dis., 73: 131-134.

Pires, C.A.A., F.O.M. Jucá Neto, N.C. de Albuquerque, G.M.M. Macedo and K.d.N.M. Batista et al., 2015. Leprosy Reactions in Patients Coinfected with HIV: Clinical Aspects and Outcomes in Two Comparative Cohorts in the Amazon Region, Brazil. PLoS Negl. Trop. Dis. DOI: 10.1371/journal.pntd.0003818

Ramos, J.M., Á.M. Costa e Silva, R.M. Martins and F.J. Dutra Souto, 2011. Prevalence of hepatitis B and C virus infection among leprosy patients in a leprosyendemic region of central Brazil. Mem. Inst. Oswaldo Cruz. 106; 106: 632-4. DOI: 10.1590/S0074-02762011000500019

Ridley, D.S. and W.H. Jopling, 1966. Classification of leprosy according to immunity. A five-group system. Int. J. Lepr Other Mycobact. Dis., 34: 255-73. PMID: 5950347

Romanini, A.M., D.S. Souza and S. Yuge, 2005. Exuberant granulomatous late secondary syphilis as differential diagnosis of reactional tuberculoid leprosy. An Bras Dermatol.

Rosa, H., A.P. Costa, M.L. Ferraz, S.C. Pedroza and A.L. Andrade et al., 1992. Association between leprosy and hepatitis B infection. A survey in Goiania, central Brazil. Rev Inst Med Trop Sao Paulo, 34: 421-6.

Sarno, E.N, X. Illarramendi, J.A. Nery, A.M. Sales and M.C. Gutierrez-Galhardo et al., 2008. HIV-M. leprae interaction: can HAART modify the course of leprosy? Public Health Rep., 123: 206-212.

Secretary of Department of STD, 2014. AIDS and Viral Hepatitis. Epidemiological Bulletin HIV/AIDS Year III - no 1 - 27a to 52a epidemiological weeks. Brasília.

Tarique, M., C. Saini, R.A. Naqvi, N. Khanna and A. Sharma et al., 2017. Molecular Immunol., 83: 72-81. DOI: 10.1016/j.molimm.2017.01.008

Teresinha Boni Ribeiro, A. and V.L. Jacociunas, 2016. The Syphilis/HIV Coinfection and its importance in the serological screening in blood banks. Clinical Biomedical Research. DOI: $10.4322 / 2357-9730.63878$

WHO, 2016. Regional Office for South-East Asia, Global Leprosy Programme. Global Leprosy Strategy accelerating towards a leprosy-free world. WHO Regional Office for South-East Asia. 